\title{
BROOKHAVEN ACCELERATOR TEST FACILITY ENERGY UPGRADE *
}

\author{
X.J. Wang " , I. Ben-Zvi, J. Sheehan and V. Yakimenko \\ BNL, Upton, NY 11973
}

\section{Abstract}

Brookhaven Accelerator Test Facility (ATF) is a laser linac complex, with a photocathode RF gun injection system and two sections of travelling wave linac. To satisfy the requirements of short wavelength free electron laser and laser acceleration experiments, ATF started an energy upgrade program with a goal of final energy on the order $100 \mathrm{MeV}$. Initial stage of the energy upgrade involves replace aged SLAC X-K5 klystron, and upgrade the magnets power supplies for the quadrupole magnets. A SLAC Energy Doubler (SLED) cavity will be installed in the second phase of the energy upgrade. Final stage of the project is to install another modulator and klystron so each section of the linac will be powered individually; which will allows us more flexibility to optimize the electron beam emittance compensation. We will also discuss issue of electron beam dynamics for operating the ATF at the higher energy.

\section{INTRODUCTION}

Brookhaven accelerator test facility (ATF) is a dedicated user facility for beam physics and radiation source R\&D. Though ATF was designed and approved for beam energy up to $120 \mathrm{MeV}$, funding constrain and resource limited ATF energy below $60 \mathrm{MeV}$. ATF is the only multi-user facility based on the photocathode RF gun technology in operation in the last decade, it typically delivered more 1000 hours user beam time annually. ATF not only provide service to industry, university and national laboratory to do beam physics research, it also actively involve many user experiments. ATF users and staff have made many significant contributions in many aspects of beam physics research. We have experimentally demonstrated emittance compensation in high field region [1], developed experimental techniques in slice emittance[2], tomography [3] and sub-picosecond timing jitters measurement[4]. We proposed and experimentally demonstrated electron beam micro-bunching in the photoinjector[5]. The photocathode RF injection system developed at ATF now is being used in many laboratories both in abroad and US. ATF users have experimentally demonstrated two laser acceleration techniques, i.e. Inverse Cerenkov Acceleration (ICA) [6] and Inverse Free Electron Laser (IFEL) [7] acceleration; Self Amplified Spontaneous Emission (SASE) was

"Work supported by U.S D.O.E contract DE-AC02-98CH10886.

"Email: xwang@bnl.gov observed at both 1 and $5 \mu \mathrm{m}[8,9]$. We have also demonstrated nano-meter resolution beam position monitors [10] and electron beam micro-bunching at optical wavelength [11].

One of the primary missions of the ATF is to explore the new high gradient acceleration techniques, specially various laser acceleration techniques. With new TW CO2 laser system, and the ATF capability of producing Picosecond to femto-second electron beams[6,11], ATF can be the host for many so called second-generation laser acceleration experiments. Pre-bunched, higher energy electron beam injected into the laser accelerators has many advantages, such as better beam quality preservation in plasma laser acceleration schemes and ICA, and longer effective interaction length.

Another type of ATF experiment demanding higher beam energy is SASE FEL and femto-second hard X-ray production by laser Compton scattering. Significant gain was measured in SASE FEL experiments [12] in IR range, but many important issues, such as transverse mode profile, longitudinal mode structure, and saturation remain to be studies if SASE process can be used to produce Xray for the fourth generation light source. Multi-institute collaboration now is constructing a Visible-Infrared SASE Amplifier (VISA) FEL experiment at the ATF to study those issues. To optimise the SASE FEL gain for the ATF beam parameters of $2.5 \mathrm{~mm}$-mrad normalize rms emittance and 200 A peak current, an undulator of period $1.8 \mathrm{~cm}$ and total length of 4 meters is required [13]. It is critical to generate visible radiation so we can take advantage of the sensitive and advanced photon beam diagnostic techniques available in the visible wavelength to study those issues. To generate SASE radiation in the $800-600 \mathrm{~nm}$ range with the above undulator, it requires 70 to $85 \mathrm{MeV}$ electron beams. For Compton scattering experiment, $100 \mathrm{MeV}$ electron beam interacts with $\mathrm{CO}_{2}$ laser $(10.6 \mu \mathrm{m})$ could produce bright sub-picosecond X-ray whose wavelength shorter than $1 \AA$.

Another important consideration for ATF energy upgrade is to optimise the emittance compensation and produce 100 femto-second or shorter electron beams. For emittance compensation photocathode RF gun injection system, linac plays two important roles. One is to boost the beam energy to reduce the space-charge effect, which decrease rapidly with the beam energy. The second function of the linac is to match the electron beam envelop and minimise the final emittance. Higher energy 


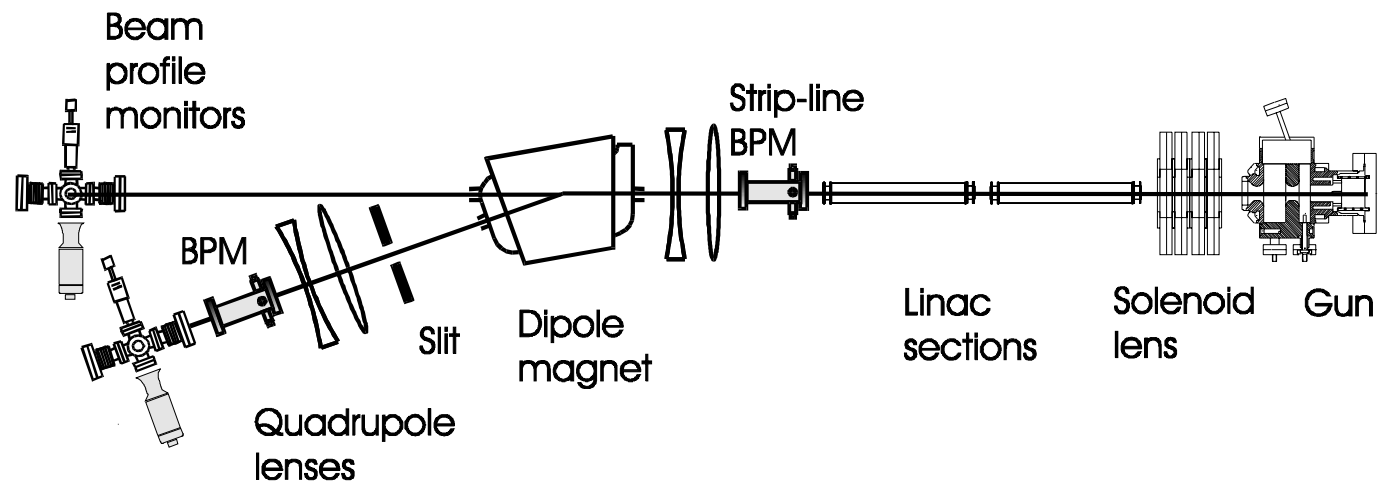

will allow more flexibility to minimise the beam emittance.

We first briefly describe the ATF photocathode RF gun injector and linac, then the strategy of ATF $100 \mathrm{MeV}$ energy up-grade and status of the project is discussed in the following section. The beam dynamic issue involve emittance compensation process will be discussed later.

\section{ATF PHOTOCATHODE RF GUN INJECTOR AND LINAC}

Fig. 1 is the schematic of the ATF photocathode RF gun injection system and linac. The photocathode RF gun injection system consists of a 1.6 cell high brightness photocathode RF gun, single solenoid magnet for emittance compensation, normal incident laser optics station, and photoelectron beam diagnostics station for electron beam charge, energy and RF phase measurements. About one meter downstream of the photocathode RF gun is the linac, which is made up by two 3-meter sections of SLAC-type travelling wave linac. The RF gun and linac were powered separately by two klystrons.

A frequency quadrupled $\mathrm{Nd}: \mathrm{YAG}$ laser, which synchronised with the ATF linac RF system, is used to drive the photocathode RF gun. It is also used to switch the ATF $\mathrm{CO}_{2}$ laser system. The $\mathrm{CO} 2$ laser system is mainly used as a power source for laser acceleration experiments and study beam photon interaction.

The klystrons used at the ATF are surplus XK-5 klystrons provide by SLAC. After remarkable more 30 years' service, the output of those klystrons now starts to decline. The output of the klystron used to power two sections of the linac was measured to be less than 15

MW. The beam energy of from two sections of the linac can be estimated by:

$$
E=10 \sqrt{2 P}
$$

Where $\mathrm{P}$ is the klystron power in $\mathrm{MW}$, and $\mathrm{E}$ is the electron beam energy in $\mathrm{MeV}$. To produce $70 \mathrm{MeV}$

electron beams, we need a $25 \mathrm{MW}$ klystron. The klystron tube we selected is $30 \mathrm{MW}$ Triton klystron 8840, which is compatible with the XK-5 klystron assembly. The second phase of the ATF energy up-grade is to install a SLAC Energy Doubler (SLED) [14] cavity purchased

from China IHEP some times ago. SLED cavity give us more energy, but also make ATF linac operation more complex. To take advantage of the SLED cavity, we also installed more capacitors in the linac modulator to increase the RF pulse width. ATF magnets are capable of delivering $100 \mathrm{MeV}$ electron beam with new 30 A power supplies.

The final stage of the ATF energy upgrade involves building a new modulator and installing a new klystron, which allows us more flexibility of to minimise the electron beam emittance.

\section{STATUS OF THE ATF 100 MEV ENERGY UPGRADE}

The initial stage of the ATF energy upgrade was implemented in the late 1998. The 30 MW Triton klystron 8840 tube was installed and conditioned in the October of 1998. Fig.2 is the maximum electron beam energy as a function of the modulator charging power supply voltage. With beam energy more than $70 \mathrm{MeV}$, VISA experiment can start the experiment at $800 \mathrm{~nm}$ even without the SLED cavity installed.

More capacitors were also installed to achieve the RF pulse width more than $3 \mu$ s (Fig.3). The SLED cavity and its water system were tested. From RF measurement data (Fig.4), the Q of the SLED cavity is better than $10^{5}$, which agrees with the design values [14]. From fig.4 of the reference 14 we concluded that, the energy multiple factor of the ATF SLED cavity is about 1.5 , which will be able to produce $100 \mathrm{MeV}$ electron beam.

The last stage of the ATF energy upgrade program is to build a new modulator and klystron, so each section of the linac will be powered individually by a single klystron. This will allow us more flexibility in electron beam emittance and bunch length optimisation. If funding 
available, we expect the new modulator and klystron come on-line at the end of year 2000.

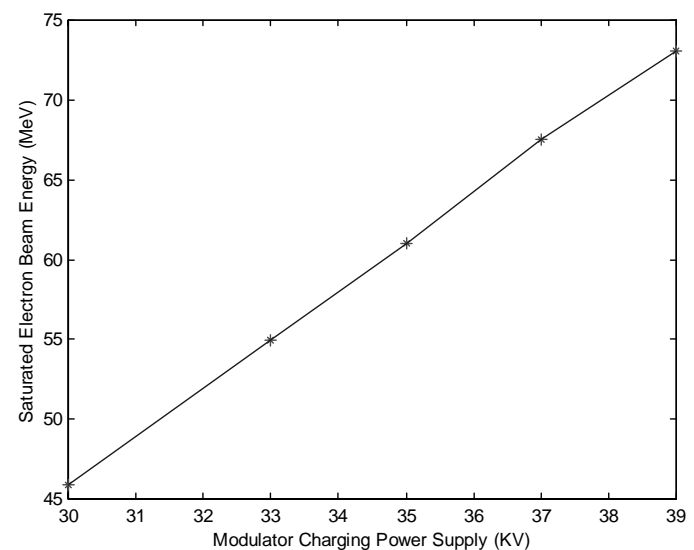

Figure 2: Electron beam energy as function of the linac modulator charging voltage.

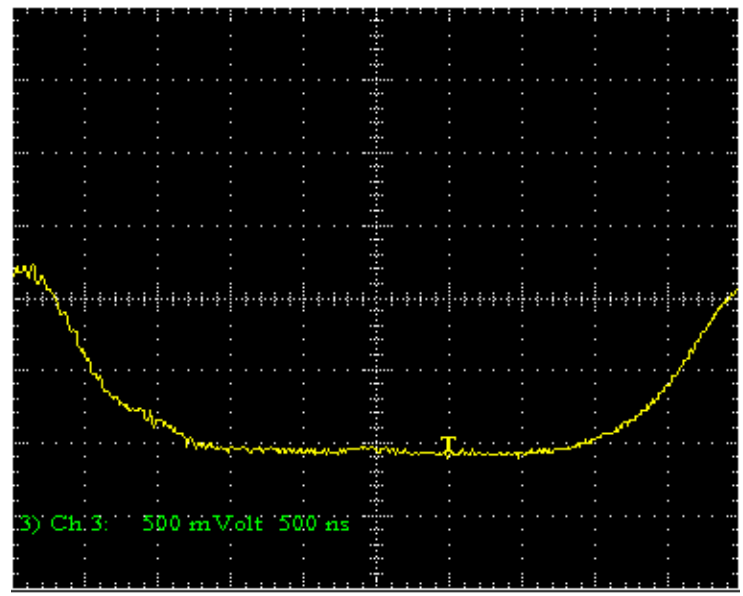

Figure 3: The linac RF pulse width measurement.

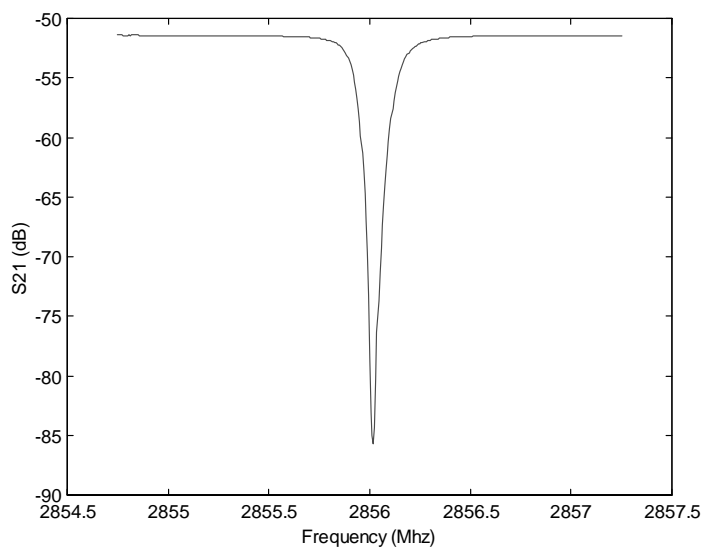

Figure 4: The SLED cavity RF measurement.

\section{BEAM MATCHING}

For a photocathode RF gun operating at emittance compensation mode [15], the acceleration gradient of the linac and final energy of the beam are critical in minimising the emittance of the photoelectron beam. For normal ATF operation, laser spot size on the cathode, laser pulse length and field gradient on the cathode and electron beam charge are fixed parameters while solenoid magnet strength was adjusted to optimise the beam emittance. With higher accelerating gradient (up to 16 $\mathrm{MV} / \mathrm{m}$ ) in the linac, we will have more freedom to optimise the final beam emittance. The procedure using so called invariant envelop theory [16] proposed by Serafini et al [17] to optimise the ATF type injector can give some guidance in general. But there are two import experimental facts the theory does not considered. We have observed both in simulation[18] and experiment [5] that, significant electron beam micro-bunching occurred for a modest amount charge $(<1 \mathrm{nC})$ situation. This is crucial for the ATF experiments (such as VISA, high gain harmonic generation FEL) which require high peak current since ATF photocathode RF gun driving laser can only produce $10 \mathrm{ps}$ (FWHM) pulselength. Another important issue was not address in the theory is the coupling we observed between the transverse emittance and electron beam bunch length [18]. Micro-bunched electron will also suffer less emittance growth due to the wakefield.

\section{REFERENCES}

[1]X.J. Wang et al, Proc. Of 1995 Part. Accel. Conf., p.890 -892.

[2] X. Qiu et al, Phys. Rev. Lett. 76, 3723 (1996).

[3] V. Yakimenko et al, to be published in the Proc. Of EPAC98, BNL-65675 (1998).

[4] X.J. Wang and I. Ben-Zvi, AIP Conf. Proc. 390, p.232 - 239 (1996). [5] X. J. Wang et al, Phys. Rev. E 54, R3121 (1996).

[6] W. D. Kimura et al, Phys. Rev. Lett. 76, 546 (1995).

[7] A. van Steenbergen at al, Phys. Rev. Lett. 77, 2690 (1996).

[8] M. Bazien et al, Phys. Rev. E 57, 6093 (1998).

[9] L.H. Yu et al, these proceeding.

[10] Vladimir Balakin, these proceeding.

[11] Y. Liu et al, Phys. Rev. Lett. 80, 4418 (1998).

[12] M. Hogan et al, Phys. Rev. Lett. 81, 4867 (1998).

[13] R. Carr et al, to be published in the Proc. Of FEL 98.

[14] Z.D. Farkas et al, Proc. $9^{\text {th }}$ International Conf. On High Energy Accelerators, p.575 (1974).

[15] B.E. Carlsten, Nucl. Inst. Methods A 285, 313(1989).

[16] L. Serafini and J.B. Rosenzweig, Phys. Rev. E 54, R3121 (1997).

[17] L. Serafini and J.B. Rosenzweig, Proc. Of PAC 97, p.2876 (1997).

[18] X.J. Wang, UCLA Ph.D thesis, UCLA-CAA0086-2/92 (1992).

[19] X.J Wang and I. Ben-Zvi, Proc. Of PAC 97, p.2793 (1997). 